\title{
XXVIII. On the speed of signalling through heterogeneous telegraph circuits
}

\section{Oliver Heaviside}

To cite this article: Oliver Heaviside (1877) XXVIII. On the speed of signalling through heterogeneous telegraph circuits, Philosophical Magazine Series 5, 3:17, 211-221, DOI: $10.1080 / 14786447708639220$

To link to this article: http://dx.doi.org/10.1080/14786447708639220

曲 Published online: 13 May 2009.

Submit your article to this journal $[\pi$

Џ Article views: 5

Q View related articles $\asymp$ 
The following Table shows the closeness of approximation, (theoretical - observed) $\div$ observed in each of the foregoing comparisons:-

\begin{tabular}{|c|c|c|c|c|c|c|c|}
\hline $\begin{array}{l}1 . \\
2 . \\
3 . \\
4 . \\
5 . \\
6 . \\
7 . \\
8 . \\
9 . \\
19 . \\
11 . \\
12 . \\
13 . \\
14 . \\
15 .\end{array}$ & $\begin{array}{r}-.0039 \\
.0197 \\
-.0158 \\
\cdot 0109 \\
-.0014 \\
\cdot 0001 \\
.0033 \\
.0074 \\
.0000 \\
\cdot 0070^{*} \\
-.0023^{*} \\
.0168^{*} \\
.0238^{*} \\
.01319^{*} \\
.0052^{*}\end{array}$ & $\begin{array}{l}16 . \\
17 . \\
18 . \\
19 . \\
20 . \\
21 . \\
22 . \\
23 . \\
24 . \\
25 . \\
26 . \\
27 . \\
28 . \\
29 . \\
30 .\end{array}$ & 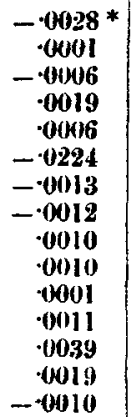 & $\begin{array}{l}31 . \\
32 . \\
33 . \\
34 . \\
35 . \\
36 . \\
37 . \\
38 . \\
39 . \\
40 . \\
41 . \\
42 . \\
43 . \\
44 .\end{array}$ & $\begin{array}{r}.0001 \\
-.0005 \\
.00112 \\
-0008 \\
.0050 \\
.0006 \\
.0000 \\
.0000 \\
\cdot 0000 \\
-0409 \\
-.0045 \\
-.0018 \\
-.0038 \\
-.0078\end{array}$ & $\begin{array}{l}45 . \\
46 . \\
47 . \\
48 . \\
49 . \\
50 . \\
51 . \\
52 . \\
53 . \\
54 . \\
55 . \\
56 . \\
57 . \\
58 .\end{array}$ & $\begin{array}{r}-.0009 \\
-.0070 \\
-.0043 \\
-0291 \\
-0016 \\
-.016 \\
-0093 \\
-00123 \\
-.0025 \\
-.0014 \\
-.0013 \\
.0030 \\
-.0051 \\
-.0008 \\
.0014\end{array}$ \\
\hline
\end{tabular}

XXVIII. On the Speed of Signalling through Heterogeneous Telegraph Cireuits. By Oliver Heaviside†.

WHEN the first trials of speed of working were made on the Anglo-Danish cable, then recently laid (September 1868), it was found that a considerably higher speed could be reached in one direction than in the other. The "line " portion of the circuit consisted of a land-line on the English side of 240 ohms resistance, then a cable of $2500 \mathrm{ohms}$ resistance and capacity 120 microfarads, and a land-line on the Danish side of 1250 ohms-all approximate. The circuit was completed through a battery of $150 \mathrm{ohms}$ at one end and a Wheatstone's receiver of $750 \mathrm{ohms}$ at the other, the circuit being worked on the earth-to-earth principle, i.e. without condensers. But although the battery and receiver at each end were the same, or nearly so, the maximum speed obtained withWheatstone's transmitter, making mechanically exact signals, was 40 per cent. higher from England to Denmark than from Denmark to England $\ddagger$. This unexpected result was abundantly contirmed by the subsequent experience of every-day practice, which proved the existence of a difference in working-speed in opposite directions varying from 20 to 40 per cent. at different times, mainly according to the state of insulation of the land-lines.

* According to Herschel.

+ Communicated by the Author.

I It may be interesting to state the actual speeds obtained on this circuit with different instruments. Morse, 60 to 75 letters per minute; Wheatstone's transmitter and receiver, 90 to 140 letters per minute; Wheatstone's transmitter and Thomson's recorder, 300 to 360 letters per minute : in all cases without condensers. 
Later on the same instruments were introduced between London and Amsterdam, on a circuit consisting of a land-line of 130 miles on the English side, then a cable of 120 miles, and on the Dutch side a land-line of 20 miles (Culley, Journ. Soc. Tel. Eng. vol, i.). In this case the maximum speed obtained was 50 per cent. higher from Amsterdam to London than vice versâ. Again, on the London-Dublin circuit, consisting of cable 66 miles and land-lines 266 and 10 miles, the longer line being on the English side, the speed from Dublin to London was double that obtained in the reverse direction, viz. 80 and 40 words per minute respectively. Similarly between London and Belfast.

In all these cases it is to be observed that the station nearest the cable receives the most slowly, and that the greater the inequality of resistance of the land-lines, the greater is the difference in the working-speeds. This seems to point directly to the conclusion that the uncentrical position of the cable in the circuit actually causes the retardation to be greater in one direction than in the other. The fact that the cable receives a much larger charge of electricity when the battery is connected to the end of the shorter than to the end of the longer landline might, on a cursory examination, seem to corroborate this conclusion. But when the light of theory is thrown upon this view of the matter it is at once found to be untenable.

It is easily shown that if condensers be distributed in any arbitrary manner along a line which is to earth at each end, dividing it into sections having any resistances, and the condensers be all initially discharged, the introduction of an electromotive force in the first section will cause the current to rise in the last section, in the same manner as the same electromotive force in the last section will cause the current to rise in the first section. Furthermore, it may be shown that if leaks be introduced on the line in any arbitrary manner, the same property will hold good. (The differential equation of the current, which is linear and of the same degree as the number of condensers, is the same for the first and last sections; and the conditions to determine the arbitrary constants are the same.) Now every telegraph-line, however irregular it may be in its resistance, capacity, and insulation in different places, may be considered as such a system of condensers and leaks, infinite in number if necessary; whence it follows that on any line there is absolutely no difference in the retardation in either direction, meaning by retardation the time required for an electromotive force at one end to cause the current at the other end to reach any stated fraction of its maximum. Therefore, to account for the facts, which cannot be gainsaid, we must 
look outside the line and fix our attention on the sending-and receiving-apparatus. The actual cause or causes must, however, be of such a nature that they only come into operation when the capacity, or the leakage, is unsymmetrically situated in the circuit. No perceptible difference in working-speed was observed on the Anglo-Danish circuit when the correspondence was maintained between the two ends of the cable itself. Now, since in all the cases described Wheatstone's transmitter was employed, it is natural to inquire whether the difference is due to any peculiarity in the method of making the signals with that instrument. If so, then we need not expect any difference to exist when simple reversals are made. But, in fact, it exists even then. An instance bearing this out was described by Mr. Varley before the Submarine-Cable Committee (Sub. Report, p. 156). Experimenting with his "wave-bisector" on the underground lines between London and Liverpool, Mr. Varley found that the introduction of resistance at the battery-end of the line lowered the speed to a greater extent than its introduction at the receiving-end, where indeed it made little difference. Here the speed was inversely as the retardation, since the wave-bisector made simple reversals. Mr. Varley attributed the difference to the leakage; but this is in direct contradiction to the theoretical result, that neither leakage nor irregularity in distribution of capacity can, acting alone, cause any difference. Also the difference existed on the Anglo-Danish circuit when simple reversals were made with the transmitter, but apparently to a smaller extent. It was quite perceptible (10 or 20 per cent.) with key-sending, using a common reversing key-though the exact amount of the difference could then not be exactly estimated, since operators differ nearly as much in their handsignalling as in their hand-writing. Although, therefore, in the case of Wheatstone's transmitter the difference in workingspeed may be, and I believe is, mainly due to a peculiarity of that instrument, yet when plain reversals are sent, there must actually be a difference in the retardation in opposite directions ; and this I believe is due to the fact, which comes out on closer inspection, that it is not the same circuit which is being worked when the direction of working is reversed.

Let the line consist of a cable of resistance c, having landlines of resistances $a$ and $b$ attached to its ends, and let the battery and receiver resistances be $f$ and $g$ respectively. Then fig. 1 shows the arrangement when $A$ sends to B. Further, suppose for simplicity, and to avoid analytical calculations, that the cable's resistance is small compared with the total resistance of the circuit. Then we may obtain tolerably accu- 
rate results by considering the cable's capacity as collected at its centre. Then, by the theory of the condenser, when A ap-

Fig. 1.

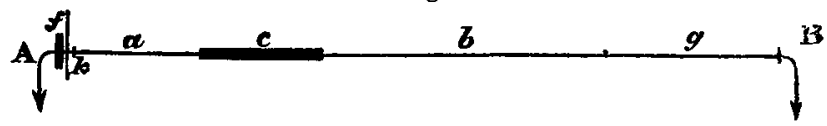

plies his battery to the line, the current rises at $B$ according to the formula

$$
\mathrm{C}=\frac{\mathrm{E}}{\mathrm{R}}\left(1-\epsilon^{-\frac{t}{\mathrm{~T}}}\right),
$$

where $\mathrm{C}$ is the current, $\mathrm{E}$ the electromotive force, $\mathrm{R}$ the total resistance between $\mathrm{A}$ and $\mathrm{B}, t$ the time, and

$$
\mathrm{T}=\frac{\mathrm{S}}{\mathrm{R}}\left(\frac{c}{2}+a+f\right)\left(\frac{c}{2}+b+g\right),
$$

where $\mathrm{S}$ is the cable's capacity. Thus the magnitude of $\mathrm{T}$ determines the slowness of the rise of the current, and we may therefore call it the retardation. (In the time $\mathrm{T}$, the current reaches abont 63 per cent. of its maximum.) Now when $B$ sends to $\mathrm{A}, f$ and $g$ change places, producing the arrangement shown in fig. 2. If $\mathrm{C}^{\prime}$ is the current $B$ produces at $A$,

Fig. 2.

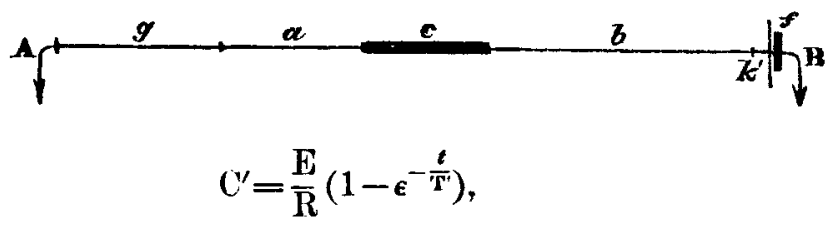

where

$$
\mathrm{T}^{\prime}=\frac{\mathrm{S}}{\mathrm{R}}\left(\frac{c}{2}+a+g\right)\left(\frac{c}{2}+b+f\right) .
$$

Comparing the values of $T$ and $\mathrm{T}^{\prime}$, we shall find that if $a=b$, $\mathrm{T}=\mathrm{T}^{\prime \prime}$; also if $f=g, \mathrm{~T}=\mathrm{T}^{\prime}$; but if $a<b$, as in the figures, $\mathrm{T}<\mathrm{T}^{\prime}$ if $f<g$, and $\mathrm{T}>\mathrm{T}^{\prime}$ if $f>g$. (Or, in plain English, the retardation is the same in both directions if the land-lines have equal resistances, whatever may be the resistances of the battery and receiver; it is also the same in both directions if the battery and receiver have equal resistances, whatever may be the resistances of the land-lines; but if the resistances of the land-lines are mnequal, the retarlation is greatest when the station nearest the cable is receiving, if at the same time the 
battery is less than the receiver resistance, and least in the contrary case. Now if the battery is always in circuit, as in making signals with a reversing key, the effect of any arbitrary signals may be calculated by the same formula, and the maximum working-speed (always provided it be within the reach of the apparatus) will be least when the station nearest the cable receives, if the battery is less than the receirer resistance, and greatest in the contrary case. Generally, the more centrally the capacity is situated the greater the retardation.

The influence of leakage or faults may be readily determined in a similar manner, since the retardation is proportional to the resistance through which the charge in the cable discharges to earth. In all cases the retardation is reduced by a fault, and the more so the nearer the fault is to the centre of capacity. If a fault be introduced on the long land-line $b$, the difference of the retardation in opposite directions is the same as before as regards direction, while its percentage amount is increased. The influence of the natural leakage of the landlines is the same, since nearly all the loss will, under ordinary circumstances, take place on the long land-line. But if a fault be introduced on the short land-line, the percentage difference is reduced instead of being increased, and its direction may even be reversed.

We have thus found that on any circuit consisting of a cable with land-lines of unequal resistance at its ends, a difference in the retardation in opposite directions is necessarily introduced when the battery and receiver have not the same resistance. Suppose, in figs. 1 and $2, f=1, a=10, c=10, b=100$, $g=10$; then the retardation from A to $B$ is to the retardation from $\mathrm{B}$ to $\mathrm{A}$ as $184: 265$, i. e. 44 per cent. greater from $\mathrm{B}$ to $A$ than from $A$ to $B$; and the natural leakage of the landlines increases this difference. But with Wheatstone's transmitter the observed difference is greater than can be thus accounted for, and exists even when there is no inequality in resistance of the battery and receiver. This is due to a peculiarity in the method of making the signals with that instrument, which is at the same time the cause of two other anomalies, viz.:- -reduction of working-speed by leakage, although the retardation is thereby reduced; and increase of workingspeed by the addition of resistance, although the retardation is thereby increased. To understand this, it is necessary to examine the way the sending-end of the line is operated upon. The point $k$ in fig. 1 , or $k^{\prime}$ in fig. 2, is always connected with the positive or negative pole of the battery, or it is insulated. Currents of equal duration follow each other, alternately + and - , separated either by no interval, or by intervals equal 
to twice, fonr, or six times the time of a current*. The armature of the receiver is adjusted neutral, so as to remain on the side any current sends it to, until an opposite current reverses its position. Lines of two lengths are thus made:-a "dot" by first a + current immediately followed by a - current to terminate it, thus + - ; and a "dash" of three times the length by first a + current, then an interval of insulation for twice as long, and lastly a - current to terminate it, thus $+00-$. At a speed much below the limiting speed the sent signals are reproduced at the receiving-end without sensible alteration ; but as the speed of working is increased and the currents have not time to reach their full strength, irregularities show themselves, which increase rapidly as the length of each contact is reduced, until at length a limiting speed is reached at which some of the signals miss fire altogether. Consider the succession of signals

$$
\begin{aligned}
& a \quad b \quad d e \text { f } f g h i j \\
& +-+-+-+-0000+-+00-+-0000
\end{aligned}
$$

(illustrating a typical failure), consisting of a series of dots, followed, after an interval of insulation, by a dot, a dash, and a dot. If the receiver is adjusted so as to record the dots $a, b$, $c, d$ perfectly, the signals $g$ and $j$ will fail. $g$ will fail because the - current $e$ has time to die away during the interval of no sent current 0000 , thus making the succeeding + current $f$ too strong; and $j$ will fail because the + current $h$ has time to die away during the interval of no sent current 00 , thus making the - current $i$ too strong. In the first case the dot is continued on to the dash, in the second the dot is lost. Thus, although generally, to get the greatest possible workingspeed, the retardation should be as small as passible, yet in this system of contacts of equal duration to make lines of unequal length, it is important that some of the currents, viz. those commencing dashes or spaces, should not die away too quickly. They are prevented from doing so, in a great measure, by the insulation of the line at the sending-end during the intervals of no sent current, which, by closing up the path at one end for the charge to escape, prolongs the current at the other. (The compensation currents, sent by an improved form of transmitter, have for their object to still further lengthen out the currents.) Now it will be seen from the figures that when $A$ insulates the line at $k$, fig. 1, the charge of the cable discharges through the resistance $\frac{c}{2}+b+g$, and that when $\mathrm{B}$ insulates at $k^{\prime}$, fig. 2 , it discharges through the smaller resistance

* Mr. Culley's 'Handbook' contains a full description of the apparalus. 
$\bar{c}+a+g$. Therefore the current dies away more quickly in the latter case, and, by reason of the before-mentioned peculiarities, the station A nearest the cable receives more slowly than $B$. The explanation of the reduction of speed by leakage is similar. The leakage lessens the retardation and consequently quickens the signals. If every signal were quickened in the same proportion, as would happen were the circuit always complete, it is evident that the speed of working must be increased ; but it is easily seen that the decrease in the retardation caused by the loss is proportionally much less when the circuit is complete than when the line is insulated at the sending-end, thus increasing the irregularity in the received signals due to the unequal intervals between the sent signals, and consequently lowering the working-speed. Again, the addition of resistance at the receiving-end, as at $A$ in fig. 2, when $B$ sends to A, may increase the working-speed. Now, since the addition of resistance obviously increases the retardation, nothing could result save a decrease of speed if the retardation of every signal were increased in the same ratio. But this is not the case ; for the retardation is increased in a greater ratio when the line is insulated at the sending-end than when the circuit is complete - exactly the opposite to what occurs with leakage: then the working-speed was lowered; now it is increased. (This reasoning will not, of course, apply to other systems of transmission.) On the other hand, the speed is lowered by inserting resistance at the sending-end, $\mathrm{B}$, fig. 2 ; for the retardation is unaltered with line insulated, and increased with complete circuit.

To ascertain the exact amount of retardation produced by resistance at either or both ends of a submarine cable, each case must be calculated separately, because the form of the curve of arrival of the current is altered, the law of the squares only holding good when exactly similar systems are compared.

\begin{tabular}{llll}
$\mathbf{A}$ & $\mathbf{B}$ & $\mathbf{C}$ & $\mathbf{D}$ \\
\hline
\end{tabular}

Let $\mathrm{BC}$ be a cable of length $l$, resistance $k$ per unit of length, capacity $c$ per unit of length; and let $A B$ and $C D$ be resistances equal to $m k l$ and $n k l$ respectively, connected to the cable at $\mathrm{B}$ and $\mathrm{C}$, and to earth at $\mathrm{A}$ and $\mathrm{D}$. Let $v$ be the potential of the conductor of the cable at distance $x$ from $\mathrm{B}$ at the time $t$. Then, according to Sir W. Thomson's theory, $v$ must satisfy

$$
\frac{d^{2} v}{d x^{2}}=c k \frac{d v}{d t}
$$


between $x=0$ and $x=l$. The general solution is

$$
v=\Sigma \mathrm{A} \sin \left(\frac{a x}{l}+b\right) \epsilon^{-\frac{a 2 t}{T}}, \quad . \quad . \quad .
$$

where $\mathrm{T}=c k l^{2}$, if $v$ vanishes for $t=\infty$. Three sets of constants, A, $a$, and $b$, have to be determined from the terminal conditions for $x$ and $t$. In A B and CD the current follows Ohm's law. Therefore

and

$$
-\frac{v}{m k l}=-\frac{1}{k} \frac{d v}{d x} \text { when } x=0,
$$

$$
\frac{v}{n k l}=-\frac{1}{k} \frac{d v}{d x} \text { when } x=l,
$$

for all values of $t$. Therefore, by (1),

and

$$
\sin b=m a \cos b, \text { or } \tan b-m a=0,
$$

or

$$
\sin (a+b)=-n a \cos (a+b) \text {, }
$$

$$
\tan (a+b)+n a=0 .
$$

Hence, eliminating $b$,

$$
\tan a=\frac{(m+n) a}{m n a^{2}-1},
$$

from which the $a$ 's can be found when $m$ and $n$ are given. The $b$ 's are already known in terms of the $a$ 's, and the A's can be found by integration if the potential of every part of the conductor of the cable is given for $t=0$. Let it be that produced by an electromotive force $\mathrm{E}$ in $\mathrm{AB}, i$. e.

then, by integration,

$$
v=\mathrm{E} \cdot \frac{l(1+n)-x}{l(1+m+n)}
$$

$$
\mathrm{A}=\frac{\frac{2 \mathrm{E} \cos b}{a}}{1+\frac{m}{1+m^{2} a^{2}}+\frac{n}{1+n^{2} a^{2}}} ;
$$

and finally, the potential at time $t$ is

$$
v=\sum_{i=1}^{i=\infty} \frac{2 \mathrm{E}}{a_{i}} \frac{\frac{1}{1+m^{2} a_{i}^{2}}}{1+\frac{m}{1+m^{2} a_{i}^{2}}+\frac{n}{1+n^{2} a_{i}^{2}}}\left(\sin +m a^{i} \cos \right) \frac{a_{i} x}{l} \epsilon^{-\frac{a_{i}{ }^{2}}{\mathrm{~T}}},
$$

from which the arrival-curres of the current may be found by making $x=l$. In the diagram six cases are shown. The 
through Heterogeneous Telegraph Circuits.

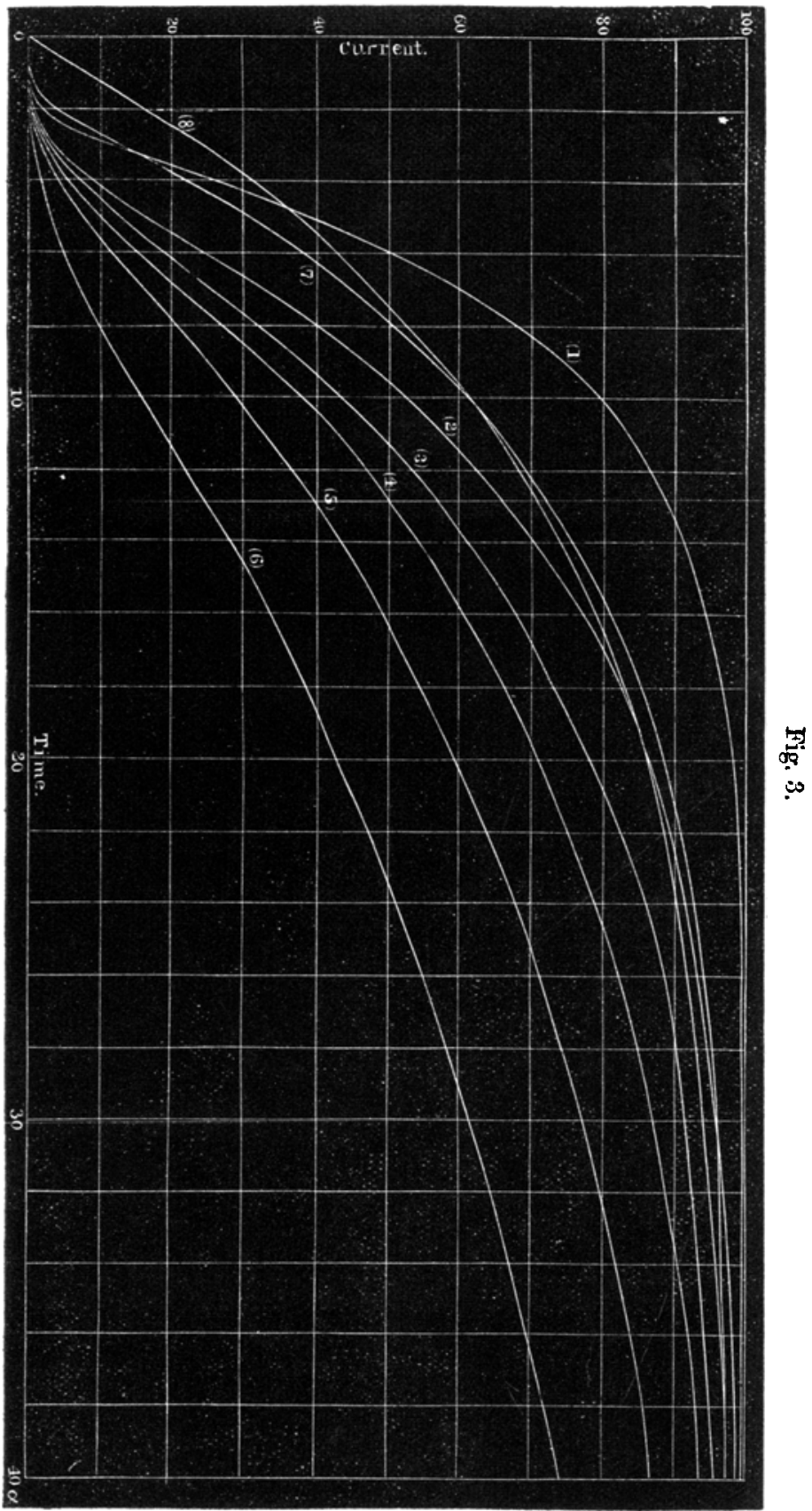


abscissas represent time, from $t=0$ to $t=40 a$, the unit being $\alpha=\frac{c k l^{2}}{10 \pi^{2}} \log , 10$. The ordinates represent the arrived current, the maximum strength being in all cases $=100$.

1. $m=0, n=0$. Let $\mathrm{N}$ be the percentage amount of received current at time $t$, then

$$
\frac{N}{100}=1+\frac{2}{\pi} \Sigma \cos i \pi \epsilon^{-\frac{i 2 \pi^{2} t}{T}}
$$

2. $m=0, n=\frac{1}{2}$.

$$
\begin{aligned}
& \tan a+\frac{a}{2}=0, \\
& \frac{\mathrm{N}}{100}=1+\sum \frac{12 \cos a}{5+\cos 2 a} \epsilon^{-\frac{a^{2} t}{\mathrm{~T}}} .
\end{aligned}
$$

3. $n=0, n=1$.

$$
\begin{aligned}
& \tan a+a=0, \\
& \frac{N}{100}=1-4 \Sigma \frac{e^{-\frac{a^{2} t}{T}}}{\cos a+\sec a} .
\end{aligned}
$$

$4 m=0, n=2$.

$$
\begin{aligned}
& \tan a+2 a=0, \\
& \frac{\mathrm{N}}{100}=1-\Sigma \frac{6 \cos a}{2+\cos a} \epsilon^{-\frac{a^{2} t}{\mathrm{~T}}} .
\end{aligned}
$$

5. $m=0, n=\infty$.

$$
\frac{N}{100}=1+\frac{4}{\pi} \Sigma \frac{\cos i \pi}{2 i-1} \epsilon^{-\frac{(2 i-1) \pi^{2} t}{4 c k r^{2}}} .
$$

6. $m=1, n=1$.

$$
\begin{aligned}
& \tan a=\frac{2 a}{a^{2}-1}, \\
& \frac{\mathrm{N}}{100}=1-\Sigma \frac{6 \epsilon^{-\frac{a^{2} t}{\mathrm{~T}}}}{3+a^{2}} .
\end{aligned}
$$

Curve (1) is the arrival-curve when no resistance is inserted at either end; curve (2) when a resistance equal to one half the cable's resistance is inserted at either end ; curve (3) when a resistance equal to the cable's is inserted at either end ; and curve (4) when twice the cable's resistance is inserted at either end. (5) shows the curve of arrival of the potential at the insulated end of a cable when the other end is raised to a con- 
stant potential ; (6) shows the arrival-curve when a resistance equal to the cable's is inserted at each end.

It will be observed from an inspection of the carves, that, when resistance is added at one end of a cable only, the effect in increasing the retardation is very great when the added resistance is small, but as more and more resistance is added there is not much further effect. The limit is reached in curve (5). But the insertion of resistance at both ends has a much greater retarding influence, which increases without limit. Compare (4) with (6): in (4) we have twice the cable's resistance at one end and none at the other ; in (6) the same resistance is equally divided at each end, and the retardation is very greatly increased.

With respect to the change in the form of the arrival-curres, it will be seen that, when resistance is inserted, the first part of the arrived current is proportionally less retarded than the later parts. Thus, comparing (1) with (6), when there is no resistance inserted the current reaches 5 per cent. of its maximum in $2.45 \alpha$, whereas (6) takes $6 \alpha$, or 2.4 times as long; to reach 10 per cent. (6) takes $3 \cdot 3$ times as long as (1); to reach 40 per cent. it takes $3 \cdot 7$ times as long, and to reach 70 per cent. 4.5 times as long.

Curves (1), (7), and (8) show the effect of different distributions of the same amount of capacity in a line of given resistance. (8) shows the arrival-curve when the capacity is all collected at the centre of the line as a single condenser, (7) when the capacity is uniformly distributed over the middle third of the line, and (1) when it is uniformly distributed over the whole length. The more the capacity is spread, the longer is the time taken for the current to reach a sensible strength, whereas the current rises rapidly the moment contact is made when the capacity is collected at one place. Curve (7) is the same as (6) with the abscissas of the latter reduced in the ratio $3: 1$; and curve (8) is the limiting form of the arrival-curve when very great equal resistances are inserted at both ends of the cable, the abscissas being reduced in the same proportion as the resistance of the circuit is increased. Its equation is

$$
\frac{N}{100}=1-\epsilon^{-\frac{4 t}{T}} .
$$

